\title{
Comparison of some different ways used for approximate solution of kinetic equation and calculations of carrier mobility
}

\author{
I.I. Boiko \\ V. Lashkaryov Institute of Semiconductor Physics, NAS of Ukraine \\ 45, prospect Nauky, 03028 Kyiv, Ukraine, \\ E-mail: igoroksanaboiko@gmail.com \\ Phone (380-44)236-5422
}

\begin{abstract}
Consideration of different problems of physical kinetics shows that obtained non-equilibrium distribution function and following kinetic coefficients calculated are significantly dependent on choice of approach to approximate solution of kinetic equation. Therefore, in real practice any separate popular way of solution cannot be confidently considered as a reliable result. Here, several approaches are used, and the obtained different distribution functions and mobility have been compared.
\end{abstract}

Keywords: kinetic equation, collision integral, mobility, standard and non-standard variants.

Manuscript received 29.08.17; revised version received 09.10.17; accepted for publication 07.12.17; published online 07.12.17.

\section{Introduction}

Theoretical investigation of kinetics is based on kinetic equation (KE) for a non-equilibrium distribution function (see [1-4]). Two fundamental problems appear from the very beginning. The first is the well-grounded deducing the kinetic equation. The second is acceptable solution of this equation and calculation of kinetic coefficients (see [5-9]). Both problems, especially the second one, are sufficiently far for the total satisfaction.

The main trouble is related with the mathematical form of $\mathrm{KE}$ for general use, which is an integerdifferential equation with specific derivatives. To find analytical precise solution of that is not possible in practice without very significant approximations. So, every time we have to find suitable approximate solution and to prove its validity. One way to improve results is to use several distinguished methods of solution and compare the obtained data. If this comparison appears efficient, we find some base to rely on obtained material.
2. Total Hamiltonian and one-particle density matrix for no equilibrium many-particle system of charged carriers

Design by the symbols $A, B$ etc. some quantum numbers that characterize states of separate particles, which makes up a system of charged band carriers. For uniform space, we assume $A \rightarrow \vec{k}_{A}$, where $\vec{k}_{A}$ is the wave vector. The values $\Psi_{A}(\vec{r})$ are basic one-particle wavefunctions. In what follows, spin variables and spin quantum numbers are not used, since any processes of spin overturn are not considered here.

One-particle density matrix is defined by the following expression:

$\rho_{A B}(t)=a_{B}^{+}(t) a_{A}(t)$.

Here, $t$ is time, $a_{A}^{+}$and $a_{A}$ are operators of generation and annihilation of band particle at the state $A$. The averaged value of shown density matrix is 
$f_{A B}(t)=\left\langle\rho_{A B}(t)\right\rangle=\left\langle a_{B}^{+}(t) a_{A}(t)\right\rangle$.

The act of averaging, we designate by angle brackets; formally this procedure is performed using a statistical operator related to total systems of band carriers and to the whole external system, represented by external microscopic accidental fields (scattering system) and macroscopic electrical field $\vec{E}$ (see [1-5]).

The base of our investigation is the set of equations for one-particle density matrices $\rho_{A B}(t)$ for nonequilibrium system of considered particles. As the start point, we used the standard motion equations for operators $\rho_{A B}(t)$ at Heisenberg representation (see, for instance [3]):

$i \hbar \frac{\partial \rho_{A B}(t)}{\partial t}=\left[\rho_{A B}(t), \hat{H}^{t o t}\right] \equiv \rho_{A B}(t) \hat{H}^{t o t}-\hat{H}^{t o t} \rho_{A B}(t)$.

One can represent the total Hamiltonian $H^{\text {tot }}$ of the whole considered system as the sum of four parts:

$\hat{H}^{t o t}=\hat{H}_{e}+\hat{H}_{S}+\hat{H}_{e S}+\hat{H}_{e e}$.

Here, the Hamiltonian $H_{e}$ concerns carriers noninteracting with microscopic scattering fields, individual Hamiltonian $H_{S}$ relates to external scattering system of impurities and phonons (see, for instance [6]) and Hamiltonian $H_{e S}=e \varphi_{S}$ describes the interaction of carriers (we call them as electrons) with this scattering system, Hamiltonian $H_{e e}$ represents $e$-e-interaction. The macroscopic electric field $\vec{E}=\left(E_{x}, 0,0\right)$. Then, the first term in right part of (2.4) is

$$
\begin{aligned}
& \hat{H}_{e}=\sum_{A B}\left(\hat{H}_{e}\right)_{A B} a_{A}^{+} a_{B}=\sum_{A B}\left[\left(\hat{H}_{e}^{(0)}\right)_{A B}+\left(\hat{H}_{e}^{(E)}\right)_{A B}\right] a_{A}^{+} a_{B}= \\
& =\sum_{A B}\left[\varepsilon_{A B} \delta_{A B}-e E_{x}(x)_{A B}\right] a_{A}^{+} a_{B} .
\end{aligned}
$$

Hamiltonian of Coulomb interaction of band carriers has the form (see [10]):

$$
\begin{aligned}
& \hat{H}_{e e}=\sum_{A B A^{\prime} B^{\prime}} V_{A B A^{\prime} B^{\prime}} a_{A}^{+} a_{A^{\prime}}^{+} a_{B^{\prime}} a_{B}, \\
& V_{A B A^{\prime} B^{\prime}}= \\
& =-\frac{e^{2}}{2 \varepsilon_{L}} \int d^{3} \bar{r} \int d^{3} \vec{r}^{\prime} \Psi_{A}^{*}(\vec{r}) \Psi_{A^{\prime}}^{*}\left(\vec{r}^{\prime}\right) \frac{1}{\left|\vec{r}-\vec{r}^{\prime}\right|} \Psi_{B^{\prime}}\left(\vec{r}^{\prime}\right) \Psi_{B}(\vec{r}) .
\end{aligned}
$$

Hamiltonian that concerns $e-S$-interaction has the form

$$
\hat{H}_{e S}=e \sum_{A B}\left(\hat{\varphi}^{(S)}\right)_{A B} a_{A}^{+} a_{B}=e \sum_{A B}\left(\hat{\varphi}^{(S)}\right)_{A B} \rho_{B A} .
$$

Below we omit the term that shows simply a shift of origin for count out the kinetic energy. As result, one obtains total Hamiltonian in the form

$$
\begin{aligned}
& \hat{H}^{\text {tot }}=\sum_{A B}\left\{\left(\hat{H}_{e}\right)_{A B}+e\left(\hat{\varphi}^{(S)}\right)_{A B}\right\} \hat{\rho}_{B A}+ \\
& +\sum_{A B A^{\prime} B^{\prime}} V_{A B A^{\prime} B^{\prime}}\left[\hat{\rho}_{B^{\prime} A}, \hat{\rho}_{B A^{\prime}}\right]_{+}+\hat{H}_{S},
\end{aligned}
$$

where $\quad[C, D]_{+}=(1 / 2)(C D+D C)$. Consider the following commutators and anticommutators:

$\left[\rho_{A B}, \rho_{M L}\right]=\delta_{A L} \rho_{M B}-\delta_{M B} \rho_{A L}$,

$\left[\rho_{A B},\left[\rho_{L^{\prime} L}, \rho_{M^{\prime} M}\right]_{+}\right]=\delta_{A L}\left[\rho_{L^{\prime} B}, \rho_{M^{\prime} M}\right]_{+}+$

$+\delta_{A M}\left[\rho_{L^{\prime} L}, \rho_{M^{\prime} B}\right]_{+}-\delta_{L^{\prime} B}\left[\rho_{A L}, \rho_{M^{\prime} M}\right]_{+}-\delta_{M^{\prime} B}\left[\rho_{L^{\prime} L}, \rho_{A M}\right]_{+}$.

Substituting (2.8) - (2.10) to (2.3) and performing necessary commutations, one can obtain the equation

$$
\begin{aligned}
& i \hbar \frac{\partial \rho_{A B}(t)}{\partial t}=\sum_{C}\left[(\hat{H})_{A C} \rho_{C B}(t)-\rho_{A C}(t)(\hat{H})_{C B}\right]+ \\
& +\sum_{C}\left\{e_{A}\left[\left(\hat{\varphi}^{(S)}(t)\right)_{A C}, \rho_{C B}(t)\right]_{+}-e_{B}\left[\rho_{A C}(t),\left(\hat{\varphi}^{(S)}(t)\right)_{C B}\right]_{+}\right\}+ \\
& +\sum_{C} \sum_{A^{\prime} B^{\prime}}\left\{V_{A C B^{\prime} A^{\prime}}\left[\rho_{A^{\prime} B^{\prime}}(t), \rho_{C B}(t)\right]_{+}-\right. \\
& \left.-V_{C B A^{\prime} B^{\prime}}\left[\rho_{A C}(t), \rho_{A^{\prime} B^{\prime}}(t)\right]_{+}\right\}
\end{aligned}
$$

Separating the density matrix $\rho_{A B}(t)$ and external scattering potential $\varphi_{S}$ into averaged values and corresponding fluctuations, one obtains the equation for the one-particle density matrix $f_{A}=\left\langle\rho_{A A}\right\rangle$ :

$\frac{\partial f_{A}}{\partial t}-\hat{\Omega}\left(f_{A}\right)=\operatorname{St} f_{A}$,

where

$$
\hat{\Omega}\left(f_{A}\right)=\sum_{C}\left[(\hat{H})_{A C} f_{C B}-(\hat{H})_{C B} f_{A C}\right](\text { at } B \rightarrow A),
$$

St $f_{A}(t)=\mathrm{St}_{e S} f_{A}(t)+\mathrm{St}_{e e} f_{A}(t)$, 
$\mathrm{St}_{e S} f_{A}(t)=(e / i \hbar) \sum_{B}\left[\left\langle\left(\left(\delta \varphi_{S}(t)\right)_{A B}, \delta \rho_{B A}(t)\right)_{+}\right\rangle-\left\langle\left(\left(\delta \varphi_{S}(t)\right)_{B A}, \delta \rho_{A B}(t)\right)_{+}\right\rangle\right] ;$

$\mathrm{St}_{e e} f_{A}(t)=(1 / i \hbar) \sum_{B A^{\prime} B^{\prime}}\left[V_{A B B^{\prime} A^{\prime}}\left\langle\left(\delta \rho_{A^{\prime} B^{\prime}}(t), \delta \rho_{B A}(t)\right)_{+}\right\rangle-V_{B A B^{\prime} A^{\prime}}\left\langle\left((\delta \rho(t))_{B^{\prime} A^{\prime}}, \delta \rho_{A B}(t)\right)_{+}\right\rangle\right]$.

\section{Matrix elements of Hamiltonian $H_{e}$}

Accept the quantum numbers $A$ as the components of wave vector $\vec{k}$ :

$A \rightarrow \vec{k}_{A}=\left(k_{A x}, k_{A y}, k_{A z}\right)$.

For the following calculations it is very convenient to use the wave functions (plane waves)

$\Psi_{A}(\vec{r})=\Psi\left(\vec{k}_{A} \vec{r}\right)=\prod_{w} \Psi\left(k_{A w} w\right)=\prod_{w} L^{-1 / 2} \exp \left(i k_{A w} w\right) ;$

here and farther $w=x, y, z$ and $-L / 2 \leq w \leq L / 2$. Every linear dimension $L$ of the system exceeds utmost every characteristic length and tends to infinity. The functions $\Psi_{A}(\vec{r})$ are proper functions for the operator of momentum $\hat{\vec{p}}=\hbar \hat{\vec{k}}$ and operator of kinetic energy $\varepsilon(\hat{\vec{p}})$ :

$-i \nabla_{w} \Psi\left(k_{A w} ; w\right)=k_{A w} \Psi\left(k_{A w} ; w\right)$,

$\nabla_{x}^{2} \Psi\left(k_{A w} ; w\right)=-k_{A w}^{2} \Psi\left(k_{A w} ; w\right)$;

at the parabolic law of dispersion

$$
\hat{\varepsilon} \Psi\left(\vec{k}_{A} ; \vec{r}\right)=\varepsilon_{A} \Psi\left(\vec{k}_{A} ; \vec{r}\right)=\left(\hbar^{2} k_{A}^{2} / 2 m\right) \Psi\left(\vec{k}_{A} ; \vec{r}\right) .
$$

Note that Hamiltonian $\hat{H}_{e}$ evidently depends on spatial coordinates (see Eq. (2.5)). In spite of all points in $\vec{r}$-space are equivalent, this Hamiltonian containing the field dependent term $\hat{H}^{(E)}$ is not arbitrary invariant in space. Therefore, a specific problem appears for collision integral. Usually, in calculations of $\mathrm{St} f_{\vec{k}}$ the field term $\hat{H}^{(E)}$ is simply omitted in collision integral (and we call that way as "standard variant", see, for instance, [2, 3] and [7-9]). In this paper, we also consider other, the so-called "non-standard variant" (see [11]), for which the field term $\left(\hat{H}^{(E)}\right)_{A C}$ in St $f_{\vec{k}}$ is retained. Farther, inside the collision integral we use the designation

$$
\left(\hat{H}_{e}\right)_{A B}=\left(\hat{H}^{(0)}\right)_{A B}+\chi\left(\hat{H}^{(E)}\right)_{A B} .
$$

Here,

$\chi=0$ for the standard variant,

$\chi=1$ for the non-standard variant.

Note at first that the wave functions (3.4) are invariant to the definite shift of argument $w$ on the length equal to DeBroglie wavelength $\lambda_{w}$ :

$$
\Psi_{A}\left(w+\lambda_{A w}\right)=L^{-1 / 2} \exp \left[i k_{A w}\left(w+\lambda_{A w}\right)\right]=\Psi_{A}(w),
$$

here,

$\lambda_{A w}=2 \pi / k_{A w}$.

It is easy to convince that matrix element of coordinate $w$ is proportional to the diagonal one at $\lambda / L \rightarrow 0$ :

$(w)_{A B}=\int_{-L / 2}^{L / 2} w \Psi *\left(k_{A w} ; w\right) \Psi\left(k_{B w} ; w\right) d w=(w)_{A A} \delta_{A, B}$

(here $\delta_{A, B}$ is Kronecker symbol).

Using the formulae (3.7) and (3.8) and shifting space of integration from the area $-L / 2 \leq w \leq L / 2$ to $L_{A w}(-) \leq w \leq L_{A w}(+)$, calculate the diagonal matrix element for components of the radius-vector:

$(w)_{A A}=\int_{L_{A w}(-)}^{L_{A w}(+)} w \Psi *\left(k_{A w} ; w\right) \Psi\left(k_{A w} ; w\right) d w$.

Here

$L_{A w}( \pm)= \pm L / 2+\lambda_{A w}$.

As a result, we obtain:

$(w)_{A A}=\int_{L_{A w}(-)}^{L_{A w}(+)} w \Psi *\left(k_{A w} ; w\right) \Psi\left(k_{A w} ; w\right) d w=$
$=L^{-1} \int_{L_{A w}(-)}^{L_{A w}^{(+)}} w d w=\lambda_{A w}=2 \pi / k_{A w}$

and

$(w)_{A B}=\left(2 \pi / k_{A w}\right) \delta_{A B}$. 
Represent the matrix element of Hamiltonian $\hat{H}_{e}$ by the form (see (3.5))

$\left(\hat{H}_{e}\right)_{A B}=\bar{\varepsilon}_{A} \delta_{A, B}=\left(\varepsilon_{A}-\chi \frac{2 \pi e E_{x}}{k_{A x}}\right) \delta_{A, B}$,

$\varepsilon_{A}=\hbar^{2} \vec{k}_{A}^{2} / 2 m$.

Using the designations $\vec{k}_{A} \rightarrow \vec{k}$ and $\vec{k}_{B} \rightarrow \vec{k}-\vec{q}$, one obtains the formula

$\bar{\varepsilon}_{A B}=\bar{\varepsilon}_{A}-\bar{\varepsilon}_{B} \rightarrow \bar{\varepsilon}_{\vec{k}}-\bar{\varepsilon}_{\vec{k}-\vec{q}}=\frac{\hbar^{2}}{2 m}\left(2 \vec{k} \vec{q}-q^{2}\right)+\chi \Theta(\vec{k}, \vec{q})$,

$\Theta(\vec{k}, \vec{q})=2 \pi e E_{x} q_{x} / k_{x}\left(k_{x}-q_{x}\right)$.

Now, we make some approximation. For second order over $q$ and simplifying the expression (3.16) by averaging the values, one obtains:

$\Theta(\vec{k}, \vec{q}) \rightarrow \hbar^{2} k_{x}^{(E)} q_{x} / m$,

$k_{x}^{(E)}=3 \pi e m E_{x}\left\langle k^{-2}\right\rangle / \hbar^{2}=3 \pi e E_{x} F_{-1 / 2}(\eta) / k_{\mathrm{B}} T F_{1 / 2}(\eta)$.

Here

$F_{r}(\eta)=\frac{1}{\Gamma(r+1)} \int_{0}^{\infty} \frac{w^{r} d w}{1+\exp (w-\eta)}, \quad \eta=\varepsilon_{\mathrm{F}} / k_{\mathrm{B}} T$.

As a result, we obtain the following approximate form of the equation (4.17):

$\bar{\varepsilon}_{\vec{k}}-\bar{\varepsilon}_{\vec{k}-\vec{q}}=\left(\hbar^{2} / m\right)\left[\left(k_{x}+\chi k_{x}^{(E)}\right) q_{x}+k_{y} q_{y}+k_{z} q_{z}-q^{2} / 2\right]$.

Introduce the new vector $\vec{\kappa}(\vec{k})$ :

$\vec{\kappa}(\vec{k})=\left(\kappa_{x}(\vec{k}), \kappa_{y}(\vec{k}), \kappa_{z}(\vec{k})\right)$,

here

$\kappa_{x}(\vec{k})=k_{x}+\chi k_{x}^{(E)}, \kappa_{y}(\vec{k})=k_{y}, \kappa_{z}(\vec{k})=k_{z}$.

Let $\bar{\omega}_{A}=\bar{\varepsilon}_{A} / \hbar$ and $\bar{\omega}_{A B}=\bar{\omega}_{A}-\bar{\omega}_{B}$. Then (at approximations shown before), one obtains from (3.19) and (3.21):

$$
\begin{aligned}
& \bar{\varepsilon}_{\vec{k}}-\bar{\varepsilon}_{\vec{k}-\vec{q}}=\hbar\left(\bar{\omega}_{\vec{k}}-\bar{\omega}_{\vec{k}-\vec{q}}\right)=\hbar \bar{\omega}_{\vec{k}, \vec{k}-\vec{q}}= \\
& =\left(\hbar^{2} / m\right)\left[\vec{\kappa}(\vec{k}) \vec{q}-q^{2} / 2\right] .
\end{aligned}
$$

\section{Collision integral}

Below we consider the averaged distribution function to be smooth in comparison with fluctuating values. Using the Laplace transformation (see [8]) $\xi(\omega)=\int_{0}^{\infty} \xi(t) \exp (i \omega t) d t$

$\xi(t)=\frac{1}{2 \pi} \int_{-\infty+i 0}^{\infty+i 0} \xi(\omega) \exp (-i \omega t) d \omega$,

one obtains from Eq. (2.11):

$-i \hbar \delta \rho_{A B}(t=0)+\hbar \omega \delta \rho_{A B}(\omega)=$

$=\left(\bar{\varepsilon}_{A}-\bar{\varepsilon}_{B}\right) \delta \rho_{A B}(\omega)+$

$+\left(f_{B}-f_{A}\right)\left[e\left(\delta \varphi^{(S)}\right)_{A B}+\sum_{A^{\prime} B^{\prime}} V_{A B B^{\prime} A^{\prime}} \delta \rho_{A^{\prime} B^{\prime}}(\omega)\right]$.

Let us introduce the following designations:

$\delta \rho_{A B}^{(0)}(\omega)=\frac{i \rho_{A B}(t=0)}{\omega-\bar{\omega}_{A B}+i 0}, M_{A B}(\omega)=\frac{f_{B}-f_{A}}{\hbar\left(\omega-\bar{\omega}_{A B}+i 0\right)}$.

Then, we find the lowest terms in the set of perturbations theory:

$\delta \rho_{A B}(\omega)=\delta \rho_{A B}^{(0)}(\omega)+$

$+M_{A B}(\omega)\left[e\left(\delta \varphi^{(S)}(\omega)\right)_{A B}+\sum_{A^{\prime} B^{\prime}} V_{A B B^{\prime} A^{\prime}} \delta \rho_{A^{\prime} B^{\prime}}^{(0)}(\omega)\right]$.

In agreement with uniformity of time, the correlator of fluctuations $\delta \varphi^{(S)}(\omega)$ can be represented as follows:

$$
\begin{aligned}
& \left\langle\delta \varphi_{A B}^{(S)}(\omega), \delta \varphi_{B A}^{(S)}\left(\omega^{\prime}\right)\right\rangle=2 \pi \delta\left(\omega+\omega^{\prime}\right)\left\langle\delta \varphi_{S}^{2}\right\rangle_{\omega}^{A B}= \\
& =\delta_{A B}(2 \pi)^{-6} \int d^{3} \vec{q} \int d^{3} \vec{q}^{\prime}\left(b_{\vec{q}}\right)_{A B}\left(b_{\vec{q}^{\prime}}\right){ }_{B A} \times \\
& \quad \times\left\langle\delta \varphi^{(S)}(\omega, \vec{q}), \delta \varphi^{(S)}\left(\omega^{\prime}, \vec{q}^{\prime}\right)\right\rangle= \\
& =\delta_{A B}(2 \pi)^{-2} \delta\left(\omega+\omega^{\prime}\right) \int d^{3} \vec{q}\left|\left(b_{\vec{q}}\right)_{A B}\right|^{2}\left\langle\delta \varphi_{S}^{2}\right\rangle_{\omega, \vec{q}},
\end{aligned}
$$

where

$\left(b_{\vec{q}}\right)_{A B}=\int \Psi_{A}^{*}(\vec{r}) \exp (i \vec{q} \vec{r}) \Psi_{B}(\vec{r}) d^{3} \vec{r}$.

In the second order of simplified theory of perturbation (see, for instance, [5])

$\left\langle\delta \rho_{A B}(t=0) \delta \rho_{A^{\prime} B^{\prime}}(t=0)\right\rangle \rightarrow \delta_{A^{\prime} B} \delta_{A B^{\prime}} f_{B}\left(1-f_{A}\right)$.

As a result, the collision integral for equilibrium external scattering system has the form $\left(A \rightarrow \vec{k}_{A}\right.$ and $\left.B \rightarrow \vec{k}_{B}\right)$ 
$\mathrm{St}_{e S} f_{A}=-\frac{e^{2}}{8 \pi^{3} \hbar^{2}} \int d^{3} \vec{k}_{B}\left\langle\delta \varphi_{S}^{2}\right\rangle_{\bar{\omega}_{A B}, \vec{k}_{A}-\vec{k}_{B}}\left\{\left[f_{A}\left(1-f_{B}\right)+f_{B}\left(1-f_{A}\right)\right] \tanh \left(\frac{\hbar \bar{\omega}_{A B}}{2 k_{\mathrm{B}} T}\right)+f_{A}-f_{B}\right\}$.

For elastic scattering

$\left\langle\delta \varphi_{S}^{2}\right\rangle_{\bar{\omega}_{A B}, \vec{k}_{A}-\vec{k}_{B}}=\left\langle\delta \varphi_{S}^{2}\right\rangle_{\vec{k}_{A}-\vec{k}_{B}} \delta\left(\bar{\omega}_{\vec{k}_{A}, \vec{k}_{B}}\right)$,

then it follows from Eq. (4.7):

$\mathrm{St}_{e S} f_{A}=-\frac{e^{2}}{8 \pi^{3} \hbar^{2}} \int d^{3} \vec{k}_{B}\left(f_{A}-f_{B}\right)\left(\delta \varphi_{S}^{2}\right)_{\vec{k}_{A}-\vec{k}_{B}} \delta\left(\bar{\omega}_{\vec{k}_{A}, \vec{k}_{B}}\right)$.

Collision integral for $e$-e-scattering can be presented by the form

$\mathrm{St}_{e e} f_{A}=\frac{4 e^{4}}{\hbar^{2} L^{3}} \sum_{B A^{\prime} B^{\prime}} \int \frac{d^{3} \vec{q}}{q^{4}\left|\varepsilon\left(\bar{\omega}_{A B}, \vec{q}\right)\right|^{2}}\left|\left(b_{\bar{q}}\right)_{A B}\right|^{2}\left|\left(b_{\vec{q}}\right)_{A^{\prime} B^{\prime}}\right|^{2} \times$ $\times \delta\left(\bar{\omega}_{A B}-\bar{\omega}_{A^{\prime} B^{\prime}}\right) \times$

$\times\left[f_{B}\left(1-f_{A}\right) f_{A^{\prime}}\left(1-f_{B^{\prime}}\right)-f_{A}\left(1-f_{B}\right) f_{B^{\prime}}\left(1-f_{A^{\prime}}\right)\right]$.

Show several correlators for different external scattering potentials (see Refs. $[5,6]$ ). They have the simple forms

$\left\langle\delta \varphi_{S}^{2}\right\rangle_{\vec{q}}=\Phi_{(S)} / q^{a} \quad(a=4$ or 2 or 0$)$.

For the system of charged impurities with concentration $n_{I}$

$\Phi_{(C I)}=32 \pi^{3} e^{2} n_{I} \vartheta\left(q-q_{0}\right) / \varepsilon_{L}^{2} ;(a=4)$

for piezoelectric scattering by longitudinal acoustic phonons

$\Phi_{(\Pi)}=G k_{\mathrm{B}} T \quad(a=2) ;$

at high temperature $\left(\hbar \omega_{p h}<<k_{\mathrm{B}} T\right)$ for quasi-ellastical scattering by polar optical phonons one can use the expression

$\Phi_{(O p t)}=8 \pi^{2} k_{\mathrm{B}} T / \varepsilon^{*} \quad(a=2) ;$

at quasi-elastic scattering on acoustic phonons $\left(\hbar s q<<k_{\mathrm{B}} T\right)$

$$
\Phi_{(A c)}=2 \pi E_{A}^{2} k_{\mathrm{B}} T / e^{2} \rho s^{2} \quad(a=0) ;
$$

for neutral impurities (see [13])

$\Phi_{(N I)}=8 \pi^{5} e^{2} r_{B}^{4} n_{(D)}^{(3)}\left[1+\exp \left(-\eta+\eta_{D}\right)\right]^{-1} \quad(a=0)$

(here $\eta_{D}=\varepsilon_{D} / k_{\mathrm{B}} T$ and $\varepsilon_{D}<0$ represents the energetic level of a donor).

Consider the static kinetic equation. Then it has the form (see Eq. (2.12))

$\frac{e}{\hbar} \vec{E} \frac{\partial f_{\vec{k}}}{\partial \vec{k}}=\mathrm{St}_{e S} f_{\vec{k}}+\mathrm{St}_{e e} f_{\vec{k}}$.

To find the approximate solution of presented kinetic equation we consider three different ways.

The first method of approach. Here we accept to consideration only the carriers scattering with small transfer of wave vector: $\langle q\rangle<\langle\langle k\rangle$. By this way we obtain Fokker-Plank equation. This situation is essentially typical for band carriers scattering by charged impurities.

The second method of approach. By this way one uses the distribution function in the form

$f_{\vec{k}} \equiv f(\vec{k})=f^{0}(\vec{k})+f_{1}(\vec{k})=f^{0}(\vec{k})+g(k) \vec{k} \vec{E}$,

here $f^{0}(\vec{k})$ is equilibrium distribution function. This approach is nucleus of the so-called "method of effective relaxation time" (see, for instance, [7]).

The third method of approach. This way concerns the model of non-equilibrium distribution function that has the form of Fermi-distribution with a shifted argument (see, for instance, [14]):

$f_{\vec{k}} \equiv f(\vec{k})=f^{0}\left(\vec{k}-\vec{k}^{u}\right) ;$

here the shift

$\vec{k}^{u}=m \vec{u} / \hbar$

and $\vec{u}$ is the macroscopic drift velocity of carriers:

$\vec{u}=\frac{2}{(2 \pi)^{3} n} \int \vec{v}(\vec{k}) f_{\vec{k}} d^{3} \vec{k}$ 


\section{Solution of kinetic equation in the Fokker-Plank form}

In this part (the first method of approach), we limit the scattering of carriers by different microscopic fields and neglect there $e$-e-scattering. The only possible basis of the last approximation is total absence of relaxation for total carriers momentum at the absence of external sources. Practically, we don't know which losses we obtain using mentioned simplification.

Here, we consider only the relaxation for which small transferred wave vectors $\vec{q}$ play the main role. Therefore, we use the expansions

$$
\begin{aligned}
& f_{\vec{k}-\vec{q}} \rightarrow f_{\vec{k}}-\vec{q} \frac{\partial f_{\vec{k}}}{\partial \vec{k}}+\frac{1}{2}\left(\vec{q} \frac{\partial}{\partial \vec{k}}\right)^{2} f_{\vec{k}}, \\
& \varepsilon_{\vec{k}-\vec{q}} \rightarrow \varepsilon_{\vec{k}}-\vec{q} \frac{\partial \varepsilon_{\vec{k}}}{\partial \vec{k}}+\frac{1}{2}\left(\vec{q} \frac{\partial}{\partial \vec{k}}\right)^{2} \varepsilon_{\vec{k}} .
\end{aligned}
$$

Then, the stationary kinetic equation (4.18) accepts the form:

$$
\begin{aligned}
& \frac{e}{\hbar} \vec{E} \frac{\partial f(\vec{k})}{\partial \vec{k}}=\frac{e^{2} m}{2(2 \pi)^{3} \hbar^{3}} \int \frac{\Phi_{(S)}}{q^{a}} d^{3} \vec{q} \times \\
& \times\left[\vec{q} \frac{\partial f(\vec{k})}{\partial \vec{k}}+\frac{\hbar^{2} \vec{k} \vec{q}}{m \mathrm{k}_{\mathrm{B}} T} f(\vec{k})[1-f(\vec{k})]\right] \delta\left(\vec{\kappa} \vec{q}-q^{2} / 2\right) .
\end{aligned}
$$

Carrying out in the equation (5.2) integration over $\vec{q}$, we obtain:

$$
\vec{E} \frac{\partial f(\vec{k})}{\partial \vec{k}}=\frac{e m \vec{\kappa} \Phi_{(S)} \varsigma(a)}{8 \pi^{2} \hbar^{2} \kappa^{a-1}}\left[\frac{\partial f(\vec{k})}{\partial \vec{k}}+\frac{\hbar^{2} \vec{k}}{m k_{\mathrm{B}} T} f(\vec{k})[1-f(\vec{k})]\right] .
$$

Represent farther the distribution function $f(\vec{k})$ in the form, containing equilibrium part $f_{0}(k)$ and additional non-equilibrium term $f_{1}^{(S)}(\vec{k})$ :

$$
\begin{aligned}
& f(\vec{k})=f_{0}(k)+f_{1}^{(S)}(\vec{k})= \\
& =\left[1+\exp \left(-\eta+\hbar^{2} k^{2} / 2 m k_{\mathrm{B}} T\right)\right]^{-1}+f_{1}^{(S)}(\vec{k}) .
\end{aligned}
$$

The density of band carriers

$$
\begin{aligned}
& n=\frac{1}{4 \pi^{3}} \int f(\vec{k}) d^{3} \vec{k}=\frac{1}{4 \pi^{3}} \int f_{0}(\vec{k}) d^{3} \vec{k}= \\
& =\frac{\sqrt{2\left(m k_{\mathrm{B}} T\right)^{3}}}{\pi^{2} \hbar^{3}} \int_{0}^{\infty} \sqrt{w}[1+\exp (w-\eta)]^{-1} d w= \\
& =\frac{\left(m k_{\mathrm{B}} T\right)^{3 / 2}}{\sqrt{2} \pi^{3 / 2} \hbar^{3}} F_{1 / 2}(\eta) .
\end{aligned}
$$

Let electric field $\vec{E}=\left(E_{x}, 0,0\right)$. For the small $E_{x}$, the linearized over this field kinetic equation has the form

$$
\begin{aligned}
& E_{x} \frac{\partial f^{0}(k)}{\partial k_{x}}= \\
& =\frac{e m \Phi_{(S)} \varsigma(a)}{8 \pi^{2} \hbar^{2} k^{a-1}}\left[\vec{k} \frac{\partial f_{1}^{(S)}(\vec{k})}{\partial \vec{k}}+\frac{\hbar^{2} k^{2}}{m k_{\mathrm{B}} T} f_{1}^{(S)}\left[1-2 f^{0}(k)\right] .\right.
\end{aligned}
$$

where

$\varsigma(a=4)=\ln \left(q_{M} / q_{0}\right), \varsigma(a<4)=2^{4-a} /(4-a)$.

Note that linearized $F P$-equation (5.6) does not contain the vector $\vec{k}^{(E)}$ (see Eq. (3.17)). Therefore, the collision integral and kinetic equation relate here only to "standard" case (that is $\chi=0$; see (3.6)).

Note that in the general case the value and the form of non-equilibrium distribution function $f(\vec{k})$ substantially depend on specifics of scattering system $S$. Therefore, farther we mark off the symbols $f, f^{0}, f_{1}$ and other symbols by the index $(S)$.

Accept the artificial form (for the first method of approach):

$f_{1}^{(S)}(\vec{k})=\left(k_{x} / k\right) \Lambda^{(S)}(k) \rightarrow\left(K_{x} / K\right) \Lambda^{(S)}(K)$.

Here and further one uses the designations:

$\vec{K}=\vec{k} / k_{T}=\hbar \vec{k} / \sqrt{2 m k_{\mathrm{B}} T}$.

$E_{(S)}=e m^{2-a / 2} \Phi_{(S)} \zeta(a) / 2^{a / 2+2} \pi^{2}\left(k_{\mathrm{B}} T\right)^{a / 2-1} \hbar^{4-a}$.

Then, it follows from the equation (5.6):

$$
\begin{aligned}
& (2 K)^{-1} \partial \Lambda^{(S)}(K) / \partial K+\Lambda^{(S)}(K) \tanh \left(K^{2} / 2-\eta / 2\right)= \\
& =-\left(a E_{x} / 2 E_{(S)} / 2 E_{(S)}\right) K^{a-2} \cosh ^{-2}\left(K^{2} / 2-\eta / 2\right) .
\end{aligned}
$$

Suitable solution of the equation (5.11) is (at number $a>0$ )

$$
\Lambda^{(S)}(K)=-\left(E_{x} / E_{(S)}\right) K^{a} \cosh ^{-2}\left(K^{2} / 2-\eta / 2\right) .
$$

Then, the solution of linearized kinetic equation (5.6) is

(C) 2017, V. Lashkaryov Institute of Semiconductor Physics, National Academy of Sciences of Ukraine 
$f_{1}^{(S, F P)}(\vec{k})=-\left(E_{x} / E_{(S)}\right)\left(\hbar^{2} k^{2} / 2 m k_{\mathrm{B}} T\right)^{a / 2} \times$

$\times\left(k_{x} / k\right) \cosh ^{-2}\left(\hbar^{2} k^{2} / 2 m k_{\mathrm{B}} T-\eta / 2\right) \rightarrow$

$\rightarrow f_{1}^{(S, F P)}(\vec{K})=-\left(K^{a-1} K_{x} E_{x} / 2 E_{(S)}\right) \times$

$\times \cosh ^{-2}\left(K^{2} / 2-\eta / 2\right)$.

Determine the mobility $\mu^{(S)}$ by using the relation

$\mu^{(S)}=j_{x}^{(S)} / e n E_{x}=$

$=\left(\hbar / m E_{x}\right) \int k_{x} f_{1}^{(S)}(\vec{k}) d^{3} \vec{k} / \int f^{0}(k) d^{3} \vec{k}$.

It follows from here:

$\mu_{(F P)}^{(S)}=\mu_{(F P / N D)}^{(S)} M_{(F P)}(a, \eta) ;$

$\mu_{(F P / N D)}^{(S)}=\frac{4 \sqrt{k_{\mathrm{B}} T} \Gamma(2+a / 2)}{3 \sqrt{\pi m} E_{(S)}} ;$

$M_{(F P)}(a, \eta)=\frac{1}{2 \Gamma(2+a / 2) F_{1 / 2}(\eta)} \times$

$\times \int_{0}^{\infty} K^{a+3} \cosh ^{-2}\left(K^{2} / 2-\eta / 2\right) d K$.

Here $\mu_{(F P / N D)}^{(S)}$ is mobility for non-degenerated carriers (see below Fig. 1).

$\mu_{(F P / N D)}^{(S)}=\frac{4 \sqrt{k_{\mathrm{B}} T} \Gamma(2+a / 2)}{3 \sqrt{\pi m} E_{(S)}}$,

$M_{(F P)}(a, \eta)=\frac{1}{2 \Gamma(2+a / 2) F_{1 / 2}(\eta)} \times$

$\times \int_{0}^{\infty} K^{a+3} \cosh ^{-2}\left(K^{2} / 2-\eta / 2\right) d K$.

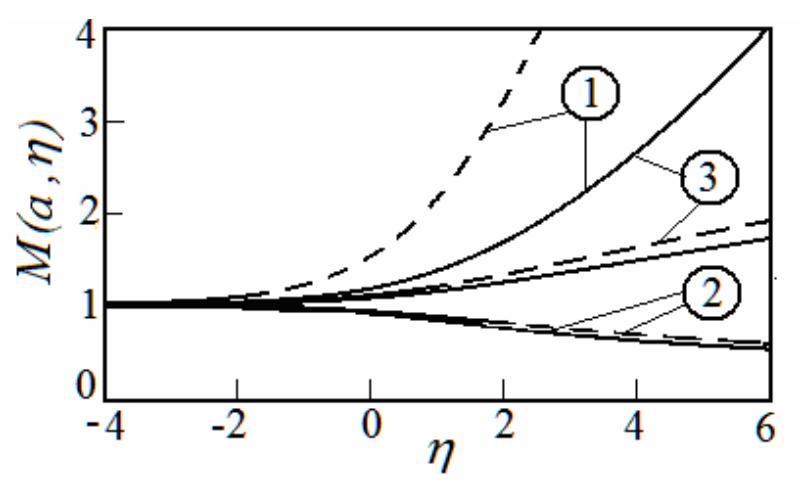

Fig. 1. - $M_{(\tau, F P)}(a, \eta),--M_{(S F D)}(a, \eta) ; a=4(1), a=0(2)$, $a=2$ (3).
Remember that Fokker-Plank approach is acceptable on practice only at sufficiently large positive parameter $a$. Note also that for small electric field the non-standard variant $(\chi=1)$ disappears.

\section{Model of effective relaxation time}

Here, we again neglect $e$-e-collisions, as that was performed before (see also remark at p.5). Using the elastic scattering and starting from the equation (4.9), we obtain the kinetic equation

$\vec{E} \frac{\partial f_{\vec{k}}}{\partial \vec{k}}=$

$=\frac{e m}{8 \pi^{3} \hbar^{2}} \int d^{3} \vec{q}\left\langle\delta \varphi_{S}^{2}\right\rangle_{\vec{q}} \delta\left(\vec{k} \vec{q}-q^{2} / 2+\chi \vec{k}^{(E)} \vec{q}\right)\left(f_{\vec{k}}-f_{\vec{k}-\vec{q}}\right)$.

Use farther the model having the form

$$
f_{\vec{k}} \equiv f(\vec{k})=f^{0}(\vec{k})+f_{1}(\vec{k})=f^{0}(\vec{k})+g^{(S)}(k) \vec{k} \vec{E} .
$$

After linearization of the equation (6.1) over the small external electrical field $E$ (see (3.17) and (4.11)), one obtains the equation for unknown function $g^{(S)}(k)$ :

$(\vec{E} \vec{k}) \frac{\partial f^{0}(k)}{\partial \varepsilon}=$

$\frac{e m^{2}}{8 \pi^{3} \hbar^{4}}\left[g_{\chi}(k)+g^{(S)}(k)\right] \vec{E} \int \Phi_{(S)} \frac{\vec{q} d^{3} \vec{q}}{q^{a}} \delta\left(\vec{k} \vec{q}-q^{2} / 2\right)$,

where

$g_{\chi}(k)=\chi \frac{3 \pi e \hbar^{2} F_{-1 / 2}(\eta)}{m k_{\mathrm{B}} T F_{1 / 2}(\eta)} \frac{\partial f^{0}(k)}{\partial \varepsilon}$

At $\vec{E}=\left(E_{x}, 0,0\right)$, it follows from here (see p. 5):

$$
\begin{aligned}
& f_{1}^{(S, \tau)}(\vec{k})= \\
& =-k_{x} E_{x}\left[\frac{\pi^{2} \hbar^{4} k^{a-1}}{m^{2} e k_{\mathrm{B}} T \Phi_{(S)} \varsigma(a)}-\chi \frac{3 \pi e \hbar^{2} F_{-1 / 2}(\eta)}{m\left(k_{\mathrm{B}} T\right)^{2} F_{1 / 2}(\eta)}\right] \times \\
& \times \cosh ^{-2}\left(\hbar^{2} k^{2} / 4 m k_{\mathrm{B}} T-\eta / 2\right)
\end{aligned}
$$

or

$$
f_{1}^{(S, \tau)}(\vec{K})=-K_{x} E_{x}\left[\frac{K^{a-1}}{4 E_{(S)}}-\frac{\chi}{E_{T}} \frac{F_{-1 / 2}(\eta)}{F_{1 / 2}(\eta)}\right] \times
$$

$\times \cosh ^{-2}\left(K^{2} / 2-\eta / 2\right)$, 
where

$$
E_{T}=m^{1 / 2}\left(k_{\mathrm{B}} T\right)^{3 / 2} / 3 \pi \sqrt{2} e \hbar .
$$

As a result, one obtains (see Figs. 1 and 2)

$\mu^{(S, \tau)}=-\frac{2^{5 / 2} \pi^{3 / 2}\left(k_{B} T\right)^{1 / 2}}{3 m^{1 / 2} F_{1 / 2}(\eta)} \times$

$\times \int K^{2}\left[\frac{K^{a-1}}{4 E_{(S)}}-\frac{\chi}{E_{T}} \frac{F_{-1 / 2}(\eta)}{F_{1 / 2}(\eta)}\right] \times$

$\times \cosh ^{-2}\left(K^{2} / 2-\eta / 2\right) d^{3} \vec{K}$;

$\mu^{(S, \tau)}(\chi=0)=-\frac{2^{5 / 2} \pi^{5 / 2}\left(k_{B} T\right)^{1 / 2}}{3 E_{(S)} m^{1 / 2} F_{1 / 2}(\eta)} \times$

$\times \int_{0}^{\infty} K^{a+3} \cosh ^{-2}\left(K^{2} / 2-\eta / 2\right) d K ;$

$\mu^{(S, F P)}=\frac{2 \pi^{5 / 2}\left(k_{B} T\right)^{1 / 2}}{3 F_{1 / 2}(\eta) m^{1 / 2} E_{(S)}} \times$

$\times \int_{0}^{\infty} K^{a+3} \cosh ^{-2}\left(K^{2} / 2-\eta / 2\right) d K$.

For standard variant considered here mobility $\mu_{(\tau)}^{(s)}(\chi=0)=\mu_{(\tau)}^{(s)}$ is presented by the form (see (5.14))

$\mu_{(\tau)}^{(S)}=$

$\frac{\left.2^{3 / 2} \pi^{3 / 2} \hbar^{3} \Gamma(a / 2+2)\right)}{3 e m^{2} \Phi_{(S)} \varsigma(a) k_{\mathrm{T}}^{1-a}} M(a, \eta)=\mu_{(\tau, N D)}^{(S)} M(a, \eta)$.

Note the following relation between distribution functions $f_{1}^{(S, \tau)}(\vec{k})$ and $f_{1}^{(S, F P)}(\vec{k})$ at $\chi=0$ (see (5.13) and (6.3)):

$f_{1}^{(S, F P)}(\vec{k})=2 f_{1}^{(S, \tau)}(\vec{k})$.

The same relation for mobilities follows from the determination (5.14) (see also [13]):

$\mu_{(F P)}^{(S)}=2 \mu_{(\tau)}^{(S)}$.

\section{Model of shifted Fermi-distribution (SFD). \\ Balance of forces}

Here, as the sufficiently simple model of nonequilibrium distribution function $f^{(S)}(\vec{k})$ we accept Fermi-function with a shifted argument (see (4.19)):

$$
\begin{aligned}
& f^{(S)}(\vec{k})=f_{0}\left(\vec{k}-\vec{k}_{u}^{(S)}\right)= \\
& =\left[1+\exp \left(\frac{\hbar^{2}\left(\vec{k}-m \vec{u}^{(S)} / \hbar\right)^{2} / 2 m-\varepsilon_{\mathrm{F}}}{k_{\mathrm{B}} T}\right)\right]^{-1} .
\end{aligned}
$$

It follows from (2.8) and (4.10):

$\frac{e}{\hbar} \vec{E} \frac{\partial f^{(S)}(\vec{k})}{\partial \vec{k}}=\frac{e^{2} m}{8 \pi^{3} \hbar^{3}} \times$

$\times \int d^{3} \vec{q} \Phi_{(S)} q^{-a} \delta\left(\vec{\kappa} \vec{q}-q^{2} / 2\right)\left\{f^{(S)}(\vec{k})-f^{(S)}(\vec{k}-\vec{q})\right\}+$ $+\mathrm{St}_{e e} f^{(S)}(\vec{k})$.

Note, at this expression we don't neglect $e$-escattering.

To find the relation between vectors $\vec{u}^{(S)}$ (drift velocity) and $\vec{E}$ (electrical field), apply to both sides of Eq. (7.2) the operator

$2(2 \pi)^{-3} \int \vec{k} d^{3} \vec{k}$

For the form (4.10), it is easy to see that

$\int \mathrm{St}_{e e} f^{(S)}(\vec{k}) \vec{k} d^{3} \vec{k}=0$.

Performing some uncomplicated transformations, we obtain the following balance of forces:

$\vec{E}+\frac{e m}{(2 \pi)^{6} \hbar^{2} n} \int f^{(S)}(\vec{k}) \vec{G}^{(S)}(\vec{\kappa}) d^{3} \vec{k}=0$,

where

$\vec{G}^{(S)}(\vec{\kappa})=\int \Phi_{(S)} q^{-a} \vec{q} \delta\left(\vec{\kappa} \vec{q}-q^{2} / 2\right) d^{3} \vec{q}$.

After integration over $\vec{q}$, one obtains (see (5.7) and (5.8)):

$\vec{G}^{(S)}(\vec{\kappa})=\left(\pi \vec{\kappa} / \kappa^{3}\right) \int_{0}^{2 \kappa} \Phi_{(S)} q^{3-a} d q=\pi \Phi_{(S)} \zeta(a) \vec{\kappa} \kappa^{1-a}$.

Using (7.4) - (7.6), we have

$\vec{E}+\frac{e m \pi \Phi_{(S)} \varsigma(a)}{(2 \pi)^{6} \hbar^{2} n} \int f^{(S)}(\vec{k}) \vec{\kappa} \kappa^{1-a} d^{3} \vec{k}=$

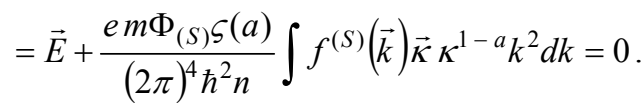

(C) 2017, V. Lashkaryov Institute of Semiconductor Physics, National Academy of Sciences of Ukraine 
The condition (7.3) does not mean that e-ecollisions do not play any role in the equation (7.7). These collisions practically have introduced their influence in the formula (7.1). In what follows, we will limit our consideration by the small external electric field $\vec{E}=\left(E_{x}, 0,0\right)$ and construct the corresponding linearized form for the non-equilibrium distribution function:

$$
\begin{aligned}
& f_{(S F D)}^{(S)}(\vec{k})=f^{0}(k)+f_{1}^{(S)}(\vec{k})= \\
& =\left[1+\exp \left(\hbar^{2} k^{2} / 2 m k_{\mathrm{B}} T-\eta\right)\right]^{-1}+ \\
& +\frac{\left(\hbar k_{x} u_{x}^{(S)} / k_{\mathrm{B}} T\right) \exp \left(\hbar^{2} k^{2} / 2 m k_{\mathrm{B}} T-\eta\right)}{\left[1+\exp \left(\hbar^{2} k^{2} / 2 m k_{\mathrm{B}} T-\eta\right)\right]^{2}} \rightarrow \\
& \rightarrow f_{(S F D)}^{(S)}(\vec{K})=f^{0}(K)+f_{1,(S F D)}^{(S)}(\vec{K})= \\
& =\left[1+\exp \left(K^{2}-\eta\right)\right]^{-1}+ \\
& +U_{x}^{(S)} K_{x} \exp \left(K^{2}-\eta\right)\left[1+\exp \left(K^{2}-\eta\right)\right]^{-2}= \\
& =\left[1+\exp \left(K^{2}-\eta\right)\right]^{-1}+ \\
& +\left(U_{x}^{(S)} K_{x} / 4\right) \cosh ^{-2}\left(K^{2} / 2-\eta / 2\right) .
\end{aligned}
$$

Here (see (3.17)),

$\vec{K}=\vec{k} / k_{T}=\hbar \vec{k} / \sqrt{2 m k_{\mathrm{B}} T} ;$

$U_{x}^{(S)}=\sqrt{2 m / k_{\mathrm{B}} T} u_{x}^{(S)}=\hbar k_{T} u_{x}^{(S)} / k_{\mathrm{B}} T ; k_{T}=\sqrt{2 m k_{\mathrm{B}} T} / \hbar ;$

$n=\frac{k_{T}^{3} F_{1 / 2}(\eta)}{4 \pi^{3 / 2}} ; K_{x}^{(E)}=\frac{k_{x}^{(E)}}{k_{T}}=\frac{6 \pi e m E_{x} F_{0}(\eta)}{3 k_{T}^{3} \hbar^{2} F_{2}(\eta)}=\frac{E_{x}}{E_{T}} ;$

$\kappa_{x} \kappa^{1-a}=k_{T}^{2-a}\left[K_{x} K^{1-a}+\chi K_{x}^{2} K_{x}^{(E)} K^{1-a}\left(\frac{1}{K_{x}^{2}}+\frac{1-a}{K^{2}}\right)\right]$.

The mobility of carriers is

$$
\begin{aligned}
& \mu_{(S F D)}^{(S)}=u_{x}^{(S)} / E_{x}=\sqrt{k_{\mathrm{B}} T / 2 m}\left(U_{x}^{(S)} / E_{x}\right)= \\
& =\left(\hbar k_{T} / 2 m\right)\left(U_{x}^{(S)} / E_{x}\right) .
\end{aligned}
$$

From the equations (7.8) and (7.11), it follows the form of linear non-equilibrium supplement for distribution function:

$$
f_{1,(S F D)}^{(S)}(\vec{K})=\frac{\mu_{(S F D)}^{(S)} \sqrt{m} E_{x} K_{x}}{\sqrt{8 k_{\mathrm{B}} T} \cosh ^{2}\left(K^{2} / 2-\eta / 2\right)} .
$$

This formula appreciably differs from the obtained before expression (6.6) (see also (6.7) and (6.8)).
Now remember that $S F D$-method, in distinction of two previous methods, allows two different variants (see [11-13]): "standard" $(\chi=0)$ and "non-standard" $(\chi=1)$. Below, at calculation of the mobility, we consider both variants.

\subsection{Standard variant $(\chi=0)$}

For this variant, one finds from (7.11):

$\kappa_{x} \kappa^{1-a}=k_{T}^{2-a} K_{x} K^{1-a}$.

Then, from (7.7) and (7.8) it follows the balance of field and dissipative forces:

$$
\begin{aligned}
& E_{x}=\frac{e m \Phi_{(S)} \zeta(a) k_{T}^{2-a} U_{x}^{(S)}}{48 \pi^{5 / 2} \hbar^{2} F_{1 / 2}(\eta)} \times \\
& \times \int_{0}^{\infty} K^{5-a} \cosh ^{-2}\left(K^{2} / 2-\eta / 2\right) d K .
\end{aligned}
$$

As a result (see (7.12)),

$$
\begin{aligned}
& \mu_{(S F D)}^{(S)}(\chi=0, T, \eta)= \\
& =\frac{12 \pi^{5 / 2} \hbar^{3}}{e \Phi_{(S)} S(a) m^{2} k_{T}{ }^{1-a} \Gamma(3-a / 2)} M_{(S F D)}(a, \eta)= \\
& =\mu_{(S F D, N D)}^{(S)}(\chi=0, T) M_{(S F D)}(a, \eta),
\end{aligned}
$$

where (see the Fig. 2)

$M_{(S F D)}(a, \eta)=2 \Gamma(3-a / 2) F_{1 / 2}(\eta) \times$

$\times\left(\int_{0}^{\infty} K^{5-a} \cosh ^{-2}\left(K^{2} / 2-\eta / 2\right) d K\right)^{-1}$,

$M_{(S F D)}(a, \eta \rightarrow-\infty) \rightarrow 1$.

The relation between mobilities calculated for three different considered methods is represented in Figs. 1 and 2. One can see that distinction of these mobilities is sufficiently evident.

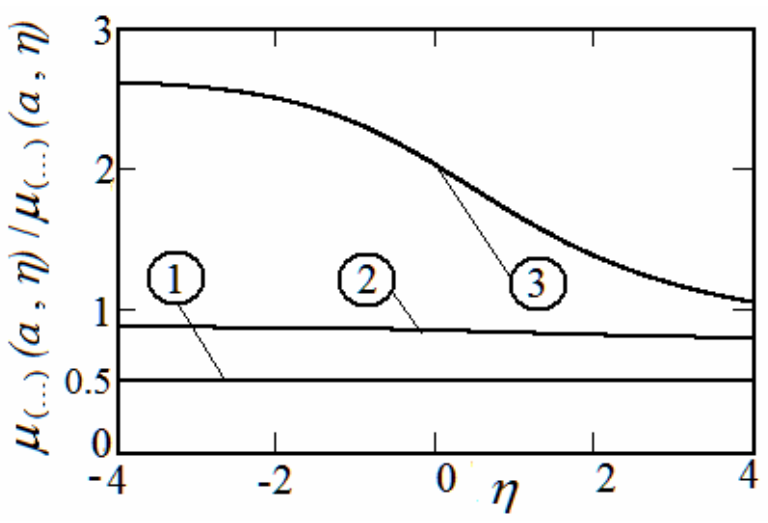

Fig. 2. $1-\mu_{(\tau)}(a, \eta) / \mu_{(F P)}(a, \eta) ; 2-\mu_{(\tau)}(a, \eta) / \mu_{(S D F)}(a, \eta)$, $a=2$ and $a=0 ; 3-\mu_{(\tau)}(a, \eta) / \mu_{(\mathrm{SDF})}(a, \eta), a=4$. 


\subsection{Non-standard variant $(\chi \neq 0)$}

Introducing the expressions (7.8) and (7.11) into the equation of balance (7.7), we obtain the following linearized form:

$$
E_{x}+\frac{e m \Phi_{(S)} \varsigma(a)}{(2 \pi)^{4} \hbar^{2} n} \int f^{(0)} \vec{\kappa} \kappa^{1-a} k^{2} d k=0
$$

or

$$
\begin{aligned}
& E_{x}=E_{(S)} U_{x}^{(S)}(\chi) \int_{0}^{\infty} \frac{K^{5-a} \exp (K-\eta) d K}{[1+\exp (K-\eta)]^{2}}+ \\
& +\chi E_{x} \xi_{(S)}(a, T, \eta)=0,
\end{aligned}
$$

where (see also (3.17), (4.7), (5.7) and (7.12))

$$
E_{(S)}=\frac{e m \Phi_{(S)} \varsigma(a) k_{T}^{2-a}}{12 \pi^{5 / 2} \hbar^{2} F_{1 / 2}(\eta)}
$$

$\xi_{(S)}(a, T, \eta)=R^{(S)}(T) \Xi(a, \eta), R^{(S)}(T)=\frac{e^{2} m^{2} \Phi_{(S)}(T)}{\hbar^{4}\left(2 k_{T}\right)^{1+a}} ;$

$\Xi(a=4, \eta)=\frac{32 F_{-1 / 2}(\eta) \ln \left(q_{M} / q_{0}\right)}{9 \pi^{3 / 2}\left[F_{1 / 2}(\eta)\right]^{2}[1+\exp (-\eta)]}$,

$\Xi(a \neq 4, \eta)=\frac{32 F_{-1 / 2}(\eta)}{9 \pi^{3 / 2}\left[F_{1 / 2}(\eta)\right]^{2}} \int_{0}^{\infty} \frac{K^{3-a} d K}{1+\exp (K-\eta)}$.

Here (see Eqs. (4.12) - (4.17) and Figs. 3 and 4),

$R^{(S)}(T)=\left(T / T_{(S)}\right)^{\gamma(S)}$

$\gamma(C I)=-5 / 2 ; \gamma(\Pi, O p t, N I)=-1 / 2 ;$

$\gamma(A c)=1 / 2 ; \gamma(N P)=3 / 2$.

Then, the mobility for non-standard variant has the form (see (7.19))

$$
\begin{aligned}
& \mu_{(S F D)}^{(S)}(\chi)=\left(\hbar k_{T} / 2 m\right)\left[U_{x}^{(S)}(\chi) / E_{x}\right]= \\
& =\mu_{(S F D)}^{(S)}(\chi=0)\left[1-\chi \xi_{(S)}(a, T, \eta)\right]
\end{aligned}
$$

or

$$
\mu_{(S F D)}^{(S)}(\chi)=\mu_{(S F D)}^{(S)}(\chi=0)-\chi \mu_{0} g(a, \eta)
$$

where (see Fig. 5)

$$
\begin{aligned}
& \mu_{0}=e \hbar / m k_{\mathrm{B}} T=2 e / \hbar k_{T}^{2}, \\
& g(a, \eta)=3 \pi^{5 / 2} M(a, \eta) \Xi(a, \eta) / 2^{a} \varsigma(a) \Gamma(3-a / 2) .
\end{aligned}
$$

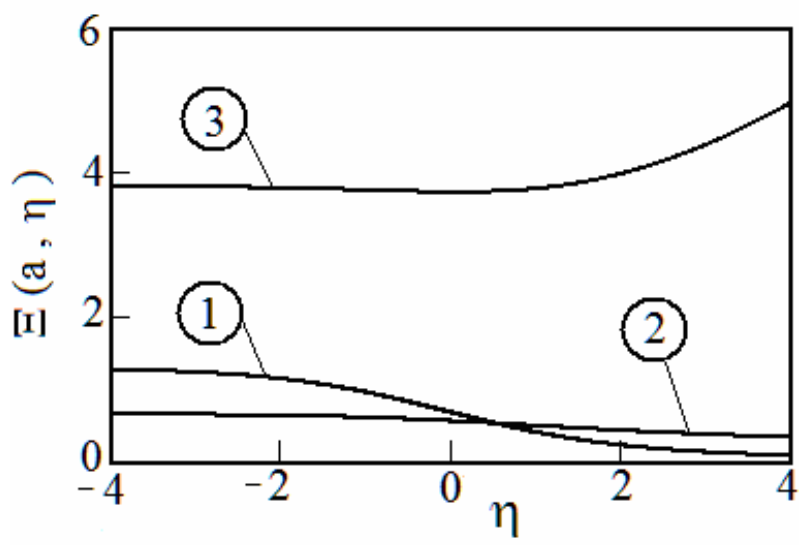

Fig. 3. $a=4(1), a=2(2), a=0$ (3).

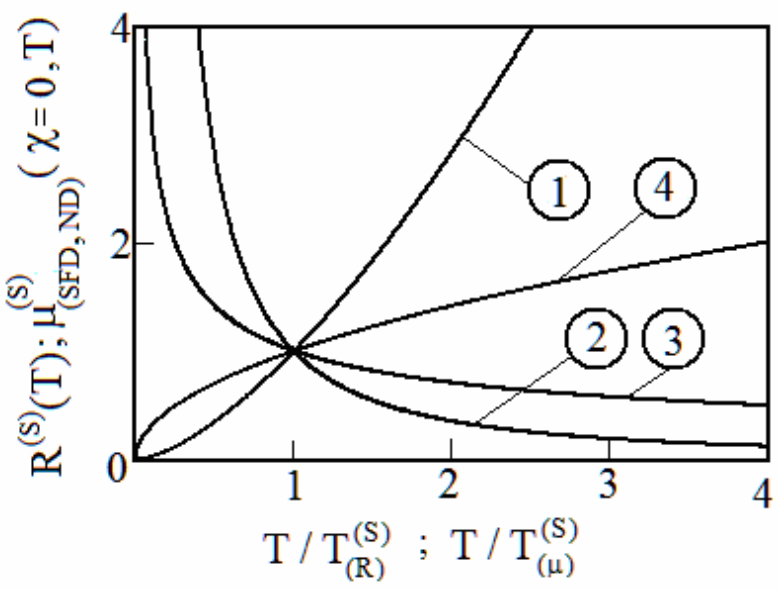

Fig. 4. $1-\left(S_{(\mu)}\right)=(\mathrm{CI}) ; 2-\left(S_{(\mu)}\right)=(A c) ; 3-\left(S_{(\mu)}\right)=\left(S_{(R)}\right)=$ $(N I, O p t, \Pi) ; 4-\left(S_{(R)}\right)=(A c)$.

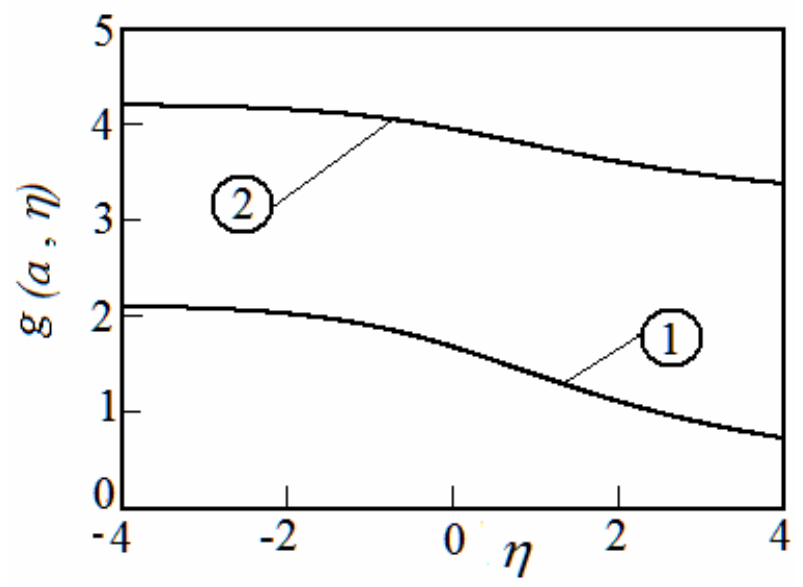

Fig. 5. $a=4(1) ; a=2, a=0$ (2).

As it follows from (7.9), (7.21) and (4.12) - (4.17): $\mu_{(S F D, N D)}^{(C I)}(\chi=0, T)=\left(T / T_{(\mu)}^{(S)}\right)^{3 / 2}$, 


$$
\begin{aligned}
& \mu_{(S F D, N D)}^{(N I, \Pi, O p t)}(\chi=0, T)=\left(T / T_{(\mu)}^{(S)}\right)^{-1 / 2} \\
& \mu_{(S F D, N D)}^{(A c)}(\chi=0, T)=\left(T / T_{(\mu)}^{(S)}\right)^{-3 / 2} \\
& \mu_{(S F D, N D)}^{(N P)}(\chi=0, T)=\left(T / T_{(\mu)}^{(S)}\right)^{-5 / 2} ; \\
& R^{(C I)}(T)=\left(T / T_{(R)}^{(S)}\right)^{-5 / 2}, R^{(N I, O p t, \Pi)}(T)=\left(T / T_{(R)}^{(S)}\right)^{-1 / 2}, \\
& R^{(A c)}(T)=\left(T / T_{(R)}^{(S)}\right)^{1 / 2}, R^{(N P)}(T)=\left(T / T_{(R)}^{(S)}\right)^{3 / 2}
\end{aligned}
$$

$$
\text { At } \xi_{(S)}(a, T, \eta)=1 \text {, the mobility } \mu_{(S F D)}^{(S)}(\chi=1)
$$

becomes zero and macroscopic movement of carriers breaks off. Only the microscopic movement of plasma is left.

Plots in Figs. 3 and 4 are calculated on the base of relations (5.7) and (7.20) - (7.28). In numerical calculations, we have taken for simplicity: $\varsigma(a=4)=\ln \left(q_{M} / q_{0}\right)=2$.

Now represent free-path $\bar{L}$ and average length of DeBroglie wave $\bar{\lambda}=\langle\lambda\rangle$ by the following forms:

$$
\begin{aligned}
& \bar{L}_{(S F D)}^{(S)}(\chi, T, \eta)=(2 m\langle\varepsilon\rangle)^{1 / 2} e^{-1} \mu_{(S F D)}^{(S)}(T, \eta)= \\
& =\frac{\hbar k_{T}(T)}{e} \sqrt{\frac{3 F_{3 / 2}(\eta)}{2 F_{1 / 2}(\eta)}} \mu_{(S F D)}^{(S)}(\chi, T, \eta), \\
& \bar{\lambda}(T, \eta)=2 \pi \sqrt{3} \sqrt{\left\langle k^{-2}\right\rangle}=\frac{2 \pi}{k_{T}(T)} \sqrt{\frac{3 F_{0}(\eta)}{2 F_{2}(\eta)}} .
\end{aligned}
$$

Then, in agreement with the expressions (7.25), (7.29) and (7.30)

$$
\frac{\bar{L}_{(S F D)}^{(S)}(\chi, T, \eta)}{\bar{\lambda}(T, \eta)}=\frac{\mu_{(S F D)}^{(S)}(\chi, T, \eta)}{\pi \mu_{0}(T)}
$$

It follows from (7.31) that mobility of carriers $\mu_{(S F D)}^{(S)}(\chi)$ reaches small value close to $\mu_{0}$, when free path $\bar{L}_{(S F D)}^{(S)}$ becomes comparable with average length of DeBroglie wave $\bar{\lambda}$.

$$
\bar{L}_{(S F D)}^{(S)}(\chi, T, \eta)=\frac{\hbar k_{T}(T)}{e} \sqrt{\frac{3 F_{3 / 2}(\eta)}{2 F_{1 / 2}(\eta)}} \mu_{(S F D)}^{(S)}(\chi, T, \eta) .
$$

\section{Discussion}

Presented results show that the obtained non-equilibrium distribution functions and following calculated mobility are distinct for different methods of solution of kinetic equation. Therefore, in real practice any obtained popular solution cannot be confidently considered as guaranteeing reliable result. One of the most interesting point is specific influence of macroscopic electric field on the collision integral with possible total disappearance of carriers mobility at characteristic situation. That appears at the non-standard variant and concerns the kinetic equation for the non-equilibrium distribution function that contains $e$-e-collisions by any way (evident or hidden). Two non-standard cases are related to the method of effective relaxation time (see Sec. 6) and to the method of distribution function having the form of Fermi-distribution with shifted argument (see Sec. 7).

\section{References}

1. Lifshits E.M. and Pitaevskiy L.P. Physical Kinetics. Moscow, Nauka, 1984 (in Russian).

2. Bogolubov N.N. Lections for Quantum Statistics. Kiev, Radianska Shkola, 1949 (in Ukrainian).

3. Bogolubov N.N. Collected works in 12 volumes, v. 5: Non-equilibrium Statistical Mechanics. Moscow, Nauka, 2006 (in Russian).

4. Klimontovich Yu.L. Statistical Physics. Moscow, Nauka, 1978 (in Russian).

5. Boiko I.I. Kinetics of Electron Gas Interacting with Fluctuating Potential. Kiev, Naukova dumka, 1993 (in Russian).

6. Anselm A.I. Introduction to Semiconductor Theory. Prentice Hall, 1982, 645 p.

7. Gantmaher V.F. and Levinson I.B. Scattering of Current Carriers in Metals and Semiconductors. Moscow, Nauka, 1984 (in Russian).

8. Bird R.B., Steward W.E., Lightfoot E.N. Transport Phenomena. John Willey \& Sons, 2007.

9. Zubarev D.N. Non-equilibrium Statistical Thermodynamics. Moscow, Nauka, 1971 (in Russian).

10. Landau L.D. and Lifshits E.M. Quantum Mechanics. Moscow, Nauka, 1963 (in Russian).

11. Boiko I.I. Dependence of the collision integral on electric field. Semiconductor Physics, Quantum Electronics \& Optoelectronics. 2015. 18, No. 2. P. 138-143.

12. Boiko I.I. Influence of field dependent form of collision integral on kinetic coefficients. Semiconductor Physics, Quantum Electronics \& Optoelectronics. 2016. 19, No. 2. P. 173-182.

13. Boiko I.I. Transport of Band Carriers in Semiconductor. Saarbruken, Palmarium Academic Publ., 2013 (in Russian).

14. Electrodynamics of Plasma, Achiezer A.I. (Ed.). Moscow, Nauka, 1974 (in Russian). 